Schmerz 2019 $33: 183-184$

https://doi.org/10.1007/s00482-019-0375-1

(c) Springer Medizin Verlag GmbH, ein Teil von Springer Nature 2019

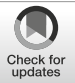

Hans-Christoph Aster · Claudia Sommer

Neurologische Klinik, Universitätsklinikum Würzburg, Würzburg, Deutschland

\title{
Chronischer Schmerz und Gesellschaft
}

[5]. Sie weisen darauf hin, dass es kurzsichtig wäre, nur ökonomische Verluste als Folge chronischer Schmerzen in unserem System zu nennen. Ein Aspekt, der bisher nämlich kaum Beachtung findet, ist der Einfluss der Gesellschaft auf chronische Schmerzen. Wie Koesling et al. aufzeigen, kann "chronischer Schmerz nicht nur als Problem für die Gesellschaft, sondern auch als Problem durch die Gesellschaft" verstanden werden. Denn die Gesellschaft mit ihren Normen und Werten, sozialen Strukturen und Ideologien kann, je nach ihrer Ausprägung und Umgang mit Schmerz und Leiden, Resilienz fördern, aber chronische Schmerzen auch verstärken. Faktoren, die hier eine Rolle spielen, sind das Spannungsfeld von einerseits Leistungsdruck und andererseits Akzeptanz sowie sozialer Unterstützung, der Zugang zu Therapiemöglichkeiten, die Lebensweise der Menschen in einer Gesellschaft und auch die Deutung der Schmerzerfahrung im sozialen Umfeld.

In experimentellen Studien wurde beispielsweise festgestellt, dass Frauen bei gleichen Bedingungen Schmerzen stärker und öfter wahrnehmen. Die Forschungsergebnisse der letzten Jahrzehnte zu diesem geschlechterspezifischen Unterschied sind allerdings inkonsistent. Studien $\mathrm{zu}$ Unterschieden in endogenen Opioiden, funktioneller Bildgebung des zentralen Nervensystems oder der Pharmakokinetik von Wirkstoffen konnten die Unterschiede nicht ausreichend erklären. Auch in diesem Bereich wurde appelliert, nicht nur nach einzelnen Mechanismen zu suchen, sondern das biopsychosoziale Modell zu berücksichtigen [8].

Die zunehmende Ruhelosigkeit unserer immer hochfrequenter funktionie- renden Gesellschaft sowie eine zunehmende Spezialisierung und Zerstückelung von Arbeitsprozessen ermöglichen eine höhere Effizienz in der Produktion, zunehmenden Wohlstand, Liberalisierung und dadurch größere individuelle Freiheit. Durch diese durchgeplante Arbeitswelt und ihre Ansprüche vermehren sich allerdings spürbar die Stressfaktoren. Wie der Soziologe Hartmut Rosa in den letzten Jahren aufzeigt, sorgt eine Verdichtung der Zeitintervalle für immer weniger Phasen in unserem Alltag, in denen wir zur Ruhe kommen und unserem Körper und Geist Zeit zur Regeneration geben könnten [7]. Hinzu kommt, dass, sobald wir die Straße betreten, ein Kampf um unsere Aufmerksamkeit beginnt. Nachrichten auf dem Smartphone, individualisierte Werbung und Neuigkeiten aus der ganzen Welt buhlen darum, unser Interesse zu erlangen. Es wäre denkbar, dass akuter Schmerz, eigentlich als Warnsignal unseres Körpers für potenzielle Gewebeschäden gedacht und mit der Interozeption, der Aufmerksamkeit für den eigenen Körper, eng verbunden, somit kaum noch seine ursprüngliche Funktion erfüllen kann, bis er stärker oder chronisch wird [3]. Spätestens dann würde er unsere Aufmerksamkeit erlangen.

Der zunehmende Wohlstand in unserer globalisierten Welt und die damit verbundenen Möglichkeiten der Selbstentfaltung lassen ein weiteres Paradoxon und einen Stressfaktor entstehen: Die "Qual der Wahl“. Die vielen Chancen und Möglichkeiten lassen manche von uns auf eine unstete Suche nach der richtigen Lebensgestaltung gehen. Ein bekannter Resilienzfaktor ist der Glaube an die Sinnhaftigkeit des eigenen Schaffens. Allerdings wird es in einer Arbeits- 
welt, in der die Aufgaben immer spezialisierter, standardisierter und damit austauschbarer gemacht werden, möglicherweise immer schwerer, seine eigene Tätigkeit im Großen und Ganzen ausmachen zu können und den Zweck einschätzen zu können. Soziologen prognostizieren, dass die Steigerung der Effektivität, zunehmende „Fließbandarbeit“ sowie das Fehlen der persönlichen Wertschätzung und Achtung durch persönlichen Umgang zu wachsender Unzufriedenheit und Stress führen könnten [1].

Der normative Druck einer kompetitiven Leistungsgesellschaft kann bewirken, dass sich Menschen immer weiter optimieren möchten, um den steigenden Anforderungen gerecht zu werden. Wenn ursprünglich zur Entspannung entwickelte Verfahren, wie die Aufmerksamkeitsmeditation, nun in großen Unternehmen angeboten werden, um die Produktionsleistung weiterhin $\mathrm{zu}$ steigern, besteht dann die Möglichkeit, dass man auch diesen Methoden ihren Nutzen nimmt? In einer Gesellschaft mit solchen Ansprüchen können Schmerzen schnell als Schwäche abgetan werden. Es besteht die Gefahr, dass Schmerzen überspielt werden, statt ihrer Ursache auf den Grund zu gehen. Nach dem zurzeit viel zitierten Motto „Fake it until you make it" versuchen manche Menschen, ihre Schmerzen zu verbergen. Andere Patienten übernehmen unbewusst gesellschaftlich akzeptierte Formen des Leidens, Koesling et al. nennen hier die Knieschmerzen eines arbeitenden Fliesenlegers als Beispiel [5], während die wahren Ursachen woanders liegen. In unserer Praxis hören wir oft Aussagen wie: „Wenn ich Krebs hätte, würden wenigstens die Menschen um mich herum mein Leiden verstehen".

Es ist durch Studien gut belegt, dass einer der wichtigsten Faktoren der Chronifizierung von Schmerzen die psychische Verfassung der Patienten ist [6]. Zu wenige Patienten in Deutschland erhalten eine adäquate Therapie, die auch ihre psychosozialen Probleme einbezieht, soziale Strukturen werden nicht ausreichend berücksichtigt. Das zeigen Umfragen, wonach $21 \%$ aller Schmerzpatienten sich gesellschaftlich isoliert fühlen [2]. Viele $\mathrm{Pa}$ tienten erzählen, dass sie sich, statt gutge- meinten Ratschlägen zuzuhören, manchmal einfach nur jemanden wünschen, der sich Zeit nimmt, ihnen zuhört und sie akzeptiert, so wie sie sind. Es ist also evident, wie wichtig der soziale Rückhalt für das Wohlergehen unserer Patienten ist. Wichtig ist, immer wieder auf diese und andere Einflüsse des gesellschaftlichen Umfelds aufmerksam zu machen, nicht zuletzt, um verengte Ansätze dazu, was „Therapie“ bedeutet, zu vermeiden. In diesem Sinne stoßen Koesling et al. eine wichtige Debatte an.

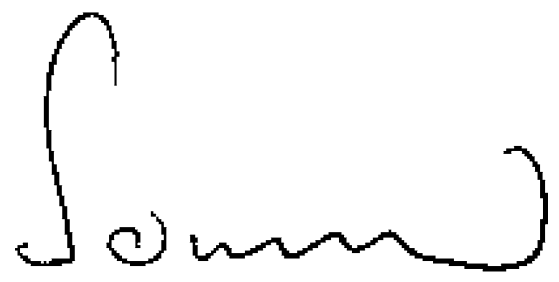

Claudia Sommer

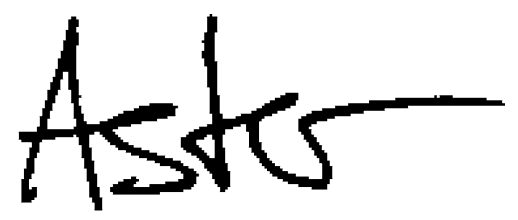

Hans-Christoph Aster

\section{Korrespondenzadresse}

Prof. Dr. Claudia Sommer

Neurologische Klinik, Universitätsklinikum

Würzburg

Josef-Schneider-Str. 11, 97080 Würzburg,

Deutschland

sommer@uni-wuerzburg.de

Interessenkonflikt. H.-C. Aster und C. Sommer geben an, dass kein Interessenkonflikt besteht.

\section{Literatur}

1. Butollo FEM, Engel T (2017) Amazonisierung der Industriearbeit? Industrie 4.0, Intralogistik und die Veränderung der Arbeitsverhältnisse in einem Montageunternehmen der Automobilindustrie. Arbeit 26(1):33-60

2. Baker Met al. Pain proposal. Improving the current and future management of chronic pain. Power Point presentation accessed at https://www.dgss org/.../Pain_Proposal_European_Consensus_ Report

3. Di Lernia D, Serino S, Riva G (2016) Pain in the body. Altered interoception in chronic pain conditions: A systematic review. Neurosci Biobehav Rev 71:328-341

4. Häuser W, Schmutzer G, Henningsen $P$ et al (2014) Chronische Schmerzen, Schmerzkrankheit und Zufriedenheit der Betroffenen mit der Schmerzbehandlung in Deutschland. Ergebnisse einer reprasentativen Bevolkerungsstichprobe. Schmerz 28:483-492

5. Koesling D, Kieselbach K, Bozzaro C (2019) Chronischer Schmerz und Gesellschaft: Soziologische Analyse einer komplexen Verschränkung. Schmerz. https://doi.org/10.1007/s00482-0190361-7

6. Landmark T, Dale O, Romundstad P et al (2018) Development and course of chronic pain over 4 years in the general population: The HUNT pain study. Eur J Pain 22:1606-1616

7. Rosa H (2005) Beschleunigung. Die Veränderung der Zeitstrukturen in der Moderne. Suhrkamp, Frankfurt

8. SchiltenwolfM, Pogatzki-ZahnEM(2015)Schmerzmedizin aus einer interkulturellen und geschlechterspezifischen Perspektive. Schmerz 29:569-575

9. Wolff R, Clar C, Lerch C et al (2011) Epidemiologie von nicht tumorbedingten chronischen Schmerzen in Deutschland. Schmerz 25:26-44 